\title{
Pregnancy after subzonal insemination with spermatozoa lacking outer dynein arms
}

\author{
J. Ph. Wolf ${ }^{1}$, D. Feneux ${ }^{1}$, D. Escalier ${ }^{1}$, D. Rodrigues ${ }^{1}$, R. Frydman ${ }^{2}$ \\ and P. Jouannet ${ }^{1}$ \\ ${ }^{1}$ Laboratoire de Biologie de la Reproduction et du Développement - Histologie, Embryologie, \\ Cytogénétique, Centre hospitalier, Université Paris XI. Bicêtre, 94275 Kremlin Bicêtre, France; and \\ ${ }^{2}$ Service de Gynécologie Obstétrique, Hôpital A. Béclère, 157, rue de la Porte de Trivaux,
} 92141 Clamart, France

\begin{abstract}
The absence of outer dynein arms in the sperm flagellum induces an abnormal movement pattern associated with male infertility. These spermatozoa can decondense in zona-free hamster oocytes but result in a very low fertilization rate in in vitro fertilization. We hypothesized that subzonal insemination could help achieve fertilization and pregnancy. A randomized prospective trial (five couples, five cycles) comparing subzonal insemination ( $n=31$ oocytes) and routine IVF ( $n=23$ oocytes) was carried out. Oocytes were microinjected with $8.5 \pm 3.6$ spermatozoa. In a second series (nine cycles), all the oocytes were microinjected with $10.5 \pm 4.3$ spermatozoa. In the randomized series, the fertilization rate was $16.1 \%$ without polyploidy, whereas no fertilization was obtained after control IVF insemination. In the second series involving nine couples, six of whom were included in the first series, the fertilization rate increased to $57.8 \%$ with a $27.8 \%$ polyspermic rate. Eightyeight per cent of the zygotes cleaved normally (29 out of 33). A total of 11 embryo transfers resulted in three pregnancies, one of which terminated one month later, a second being ongoing and the third delivering a healthy girl. A $21.4 \%$ pregnancy rate per cycle, with a $37.5 \%$ pregnancy rate per couple, justifies the use of subzonal insemination to treat this particular flagellar dyskinesia.
\end{abstract}

\section{Introduction}

Various axonemal defects have been described in the spermatozoa of infertile men. Most defects are responsible for flagellar immotility (Afzelius and Eliasson, 1979; Bacetti et al., 1979; Escalier and David, 1984; Escudier et al., 1990). In some cases, the absence of the outer dynein arms is the only detectable defect. The spermatozoa are motile, but there is a flagellar dyskinesia that is associated with male sterility; decreased sperm movement is observed. The diagnosis is based on finding less than three outer arms on all the electron micrograph axoneme cross-sections of the same sample. The movement pattern of these spermatozoa is characteristic. The shape of their principal flagellar curvature is similar to that of normal spermatozoa but their curvilinear velocity, straightline velocity and flagellar beat frequency are approximately half those of control values (Jouannet et al., 1983). These spermatozoa can migrate in cervical mucus but their penetration is partially or completely impaired (Jouannet et al., 1983). As a consequence, failure to ascend the female reproductive tract results in infertility as has been shown for other abnormal sperm movements that impede interaction between spermatozoa and cervical mucus (Aitken $e t$ al., 1986).

Received 6 May 1992.
Microfertilization techniques may be a useful treatment in cases of male infertility ( $\mathrm{Ng}$ et al., 1988; Garrisi et al., 1990; Fishel et al., 1990). Since the flagellar dyskinesia can impair the interaction between spermatozoa and zona pellucida, we hypothesized that subzonal insemination could help in achieving fertilization and pregnancy for patients whose infertility is related to this particular sperm pathology. The local Ethical Committee gave its approval. In a first series of trials, we ran a randomized clinical trial comparing a routine IVF procedure with subzonal insemination $(n=5$ cycles for five different patients). In a second series of trials, three new patients were included in the programme, and six repeated attempts were performed for patients who were already unsuccessfully involved in the first series. In view of the encouraging results of the first series, all the oocytes of the second series were microinjected with a greater number of spermatozoa without any randomization.

\section{Patients and Methods}

\section{Selection of patients}

Eight couples with primary infertility for a mean duration of 7 years (range 3-12) were included in the study. The men 

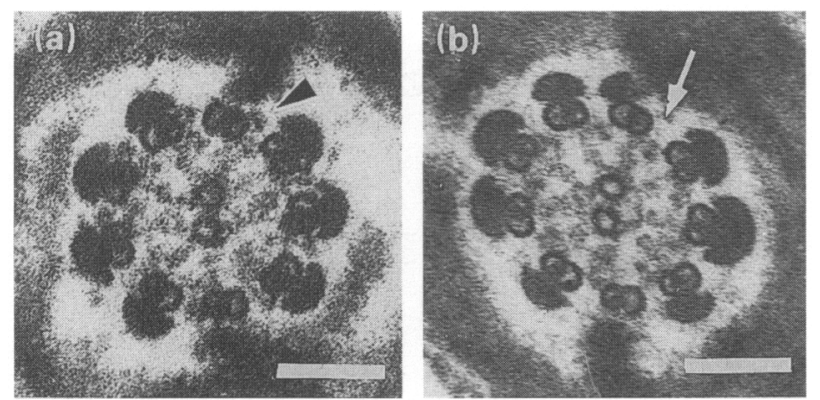

Fig. 1. Electron micrographs of the principal piece of human sperm flagella (transverse sections). (a) Normal spermatozoon exhibiting outer dynein arms (arrowhead). (b) Spermatozoon without dynein arms (arrow) from a patient. Bars $=0.1 \mu \mathrm{m}$.

were $31.5 \pm 1.5$ years old (range 29-34) and the women $30.7 \pm 2.1$ years old (range 27-34). Two couples had two previous IVF attempts each (38 oocytes) without any fertilization. Sperm counts and motility were determined by classical methods. The sperm analysis included a characterization of the sperm movement pattern using a computerized analysing system (M 2030: Hamilton Thorn, IMV France), an in vitro cervical mucus penetration and immunobead test. The sperm morphology was assessed on a smear stained with Schorr and haematoxylin according to the classification of David et al. (1975). The structural defect was suspected for these patients because their sperm exhibited a limited cervical mucus penetration test in spite of a normal or subnormal percentage of motile spermatozoa and an absence of anti-sperm antibodies. The study of their movement pattern confirmed the flagellar dyskinesia. The diagnosis was finally confirmed by examination of spermatozoa by electron microscopy, which revealed that spermatozoa from seven patients had a complete absence of outer dynein arms and $60 \%$ of spermatozoa from the last patient had this abnormality (A2) (Fig. 1). The sperm analysis was completed by a test of selection by centrifugation through a simplified Percoll gradient (95\%, 47.5\%) (Pharmacia, France) and a study of the percentage of motile forms in B2 Menezzo's medium (Bio Mérieux, France) after incubation for $24 \mathrm{~h}$ at $20^{\circ} \mathrm{C}$ (Survival Test). The sperm fusiogenic function was evaluated by a zona-free hamster oocyte penetration assay before including the patients in the programme. This test was carried out by incubating thirty eggs in a sperm suspension containing $2.5 \times 10^{6}$ motile spermatozoa $\mathrm{ml}^{-1}$ for $3 \mathrm{~h}$. The spermatozoa had been collected the day before the test and the motile spermatozoa selected by centrifugation through a two-density Percoll gradient ( 95 and $47.5 \%$ ) and then incubated for $18 \mathrm{~h}$ in BWW medium (Biggers et al., 1971) at $18^{\circ} \mathrm{C}$.

\section{Sperm preparation for oocyte insemination}

The sperm specimen was obtained by masturbation on the day before oocyte retrieval (Mortimer et al., 1989). Motile spermatozoa were selected by centrifugation through a simplified Percoll gradient spun for $20 \mathrm{~min}$ at $300 \mathrm{~g}$. Pellets were washed twice by centrifugation $(5 \mathrm{~min}, 600 \mathrm{~g}$ ), resuspended in $\mathrm{B} 2$ medium and kept for $16-18 \mathrm{~h}$ at $20^{\circ} \mathrm{C}$ before use.

\section{Oocyte preparation}

Follicular stimulation protocols were performed with a combination of a $\mathrm{GnRH}$ analogue and human menopausal gonadotrophin. Ovulation was induced with 5000 iu of hCG and oocytes were harvested $36 \mathrm{~h}$ later using a transvaginal ultrasound-guided procedure (Frydman et al, 1988). The cumuli were washed and individually placed in $40 \mu \mathrm{l}$ of B2 medium under equilibrated mineral oil. The cumulus cells were removed with $0.1 \%$ hyaluronidase (Type III, Sigma, St Louis, MO) in B2 medium and the eggs checked for their nuclear status on the basis of the presence or absence of the first polar body or of the germinal vesicle (Veeck, 1986).

In the first series of experiments, the microinjection technique was tested in a trial during which oocytes were randomly divided into two groups. Oocytes from the first group were inseminated by a routine IVF technique $(n=23)$, and the others had subzonal insemination $(n=31)$.

In the second series $(n=90)$, all the oocytes were microinjected. For both series, the procedure took place 6-8 h after egg retrieval, regardless of nuclear maturity unless oocytes still presented a germinal vesicle. In this latter case they were matured in vitro before insemination or subzonal insemination (7 of 147 oocytes).

\section{Sperm microinjection}

Microinjection was performed using two Narishige micromanipulators and an inverted Olympus IMT-2 microscope. Differential interference contrast optics were suitable for carrying out the procedure. Micropipettes were pulled on a vertical Narishige PB-7 puller ( $8 \mu \mathrm{m}$ diameter), and holding pipettes were prepared on a De Fonbrune microforge (Alcatel, France). Subzonal insemination was performed in $3 \mu \mathrm{l}$ of Hepes-buffered B2 medium containing $0.1 \mathrm{~mol}$ sucrose $1^{-1}$ at $\mathrm{pH} 7.4$, under equilibrated mineral oil. The oocytes were transferred onto a glass depression slide, and a $3 \mu \mathrm{l}$ of sperm suspension in B2 medium was placed on another slide. A few spermatozoa were aspirated into the microinjection pipette, flagellum first. The oocyte was then caught by the holding pipette and the zona pierced; spermatozoa were allowed to swim into the perivitelline space. Oocytes were then returned to the culture medium in an air: $5 \% \mathrm{CO}_{2}$ incubator at $37^{\circ} \mathrm{C}$. The total procedure took less than $5 \mathrm{~min}$. The mean number of spermatozoa microinjected was $8.5 \pm 3.6$ in the first series and $10.5 \pm 4.3$ in the second (range 1-20).

\section{Control IVF oocytes}

Control oocytes were inseminated after the cumuli had been dispersed by $0.1 \%$ hyaluronidase in B2 medium. They were inseminated with 5000 motile spermatozoa in $40 \mu \mathrm{l} \mathrm{B2}$ medium drops under mineral oil and kept in an air: $5 \% \mathrm{CO}_{2}$ incubator at $37^{\circ} \mathrm{C}$.

\section{Embryos}

The oocytes were checked $16-18 \mathrm{~h}$ after insemination or subzonal insemination for evidence of fertilization. Oocytes 
Table 1. Sperm parameters of patients included in the programme

\begin{tabular}{|c|c|c|c|c|c|c|c|}
\hline Patient & $\begin{array}{l}\text { Volume of } \\
\text { ejaculate } \\
\text { (ml) }\end{array}$ & $\begin{array}{l}\text { Number of } \\
\text { spermatozoa } \\
\times 10^{6} \mathrm{ml}^{-1}\end{array}$ & $\begin{array}{c}\text { Motile } \\
\text { spermatozoa } \\
(\%)\end{array}$ & $\begin{array}{l}\text { Total motile } \\
\text { sperm count } \\
\times 10^{\circ}\end{array}$ & $\begin{array}{c}\text { Normal } \\
\text { morphology } \\
(\%)\end{array}$ & $\begin{array}{c}\text { Acrosome } \\
\text { defect } \\
(\%)\end{array}$ & $\begin{array}{c}\text { Hamster egg } \\
\text { penetration test } \\
(\%)^{*}\end{array}$ \\
\hline $\mathrm{AI}$ & 3.2 & 10 & 15 & 4.8 & 58 & 20 & 31 \\
\hline A2 & 4.6 & 23 & 28 & 29.6 & 45 & 20 & 51 \\
\hline A 3 & 2.7 & 55 & 40 & 59.4 & 40 & 25 & 52 \\
\hline A4 & 2.8 & 80 & 20 & 44.8 & 34 & 25 & 71 \\
\hline A5 & 2.6 & 72 & 5 & 9.4 & 20 & 41 & 9 \\
\hline A6 & 1.9 & 65 & 40 & 49.4 & 30 & 32 & 66 \\
\hline A7 & 3.6 & 41 & 7 & 10.3 & 48 & 40 & 26 \\
\hline A8 & 3.2 & 127 & 15 & 60.9 & 43 & 17 & 93 \\
\hline Mean & $3.0 \pm 0.8$ & $59.1 \pm 36.4$ & $21.2 \pm 13.6$ & $33.6 \pm 23.1$ & $39.7 \pm 11.7$ & $27.5 \pm 9.2$ & $49.8 \pm 27.1$ \\
\hline
\end{tabular}

For each patient these values represent the mean of three analyses except for the zona-free hamster egg penetration test which was performed only once. *Percentage of oocytes with decondensed sperm heads.

Table 2. Fertilization rate in patients after subzonal and IVF insemination with spermatozoa lacking outer dynein arms

\begin{tabular}{|c|c|c|c|c|c|c|}
\hline \multirow[b]{2}{*}{ Patient } & \multicolumn{3}{|c|}{ Microinjected oocytes } & \multicolumn{2}{|c|}{ Inseminated oocytes } & \multirow[b]{2}{*}{ Pregnancy } \\
\hline & $\begin{array}{c}\text { Number of } \\
\text { oocytes }\end{array}$ & Fertilized & Polyspermic & $\begin{array}{c}\text { Number of } \\
\text { oocytes }\end{array}$ & Fertilized & \\
\hline AI & 8 & 1 & 0 & 8 & 0 & \\
\hline A2 & 10 & 2 & 0 & 4 & 0 & yes \\
\hline A3 & 7 & 2 & 0 & 6 & 0 & \\
\hline A4 & 4 & 0 & 0 & 4 & 0 & \\
\hline A.5 & 2 & 0 & 0 & 1 & 0 & \\
\hline Total & 31 & 5 & 0 & 23 & 0 & \\
\hline
\end{tabular}

exhibiting two pronuclei were considered normally fertilized. An oocyte was considered unfertilized or polyspermic when no pronucleus or more than two pronuclei, respectively, were found. Diploid zygotes were kept in culture for a further $24 \mathrm{~h}$. Regularly cleaved embryos only were transferred.

\section{Statistical analysis}

Groups of oocytes were compared by Chi squared tests. Differences were considered significant at $P<0.05$. Regression and correlation coefficients were studied using the STATWORKS package.

\section{Results}

\section{Characteristics of individual patient's spermatozoa}

Except for patient A1, sperm counts were normal (Jouannet $e t$ al., 1988); the mean was $59.1 \times 10^{6}$ spermatozoa $\mathrm{ml}^{-1}$ (range: $\left.10-127 \times 10^{6}\right)$. The percentage of motile spermatozoa was low for four patients (A1, A5, A7 and A8). The percentage of spermatozoa with normal morphology and the percentage with acrosome defects were comparable to those of a fertile population except for patients A5 and A7 who had an increased incidence of acrosome defects ( 41 and $40 \%$, respectively). Patient A5 also presented a reduced percentage of oocytes with decondensed sperm heads in the zona-free hamster oocyte penetration test $(9 \%)$, whereas the mean for all patients was $49.8 \pm 27.1 \%$ (Table 1). All patients had positive survival tests and no anti-sperm antibodies. Analysis of sperm movement revealed the characteristic pattern of spermatozoa lacking outer dynein arms: reduced mean straight line velocity of $5.5 \pm 14.9 \mu \mathrm{m} \mathrm{s}^{-1}$ (range 7.8-21) and normal mean lateral head displacement of $2.6 \pm 1.3 \mu \mathrm{m}$ (range $0.8-3.7$ ).

\section{Results of subzonal insemination}

A total of 147 oocytes were retrieved in 14 cycles. Sixty-eight per cent were at metaphase II at the moment of insemination or subzonal insemination. One hundred and twenty-four were microinjected. Cellular damage occurred in three cases $(2.5 \%)$.

In the randomized series, fertilization was obtained for three of the five couples by subzonal insemination (Table 2). Five oocytes out of 31 were fertilized (16.1\%). Four eggs cleaved normally and one remained blocked at the pronucleus stage. 
Table 3. Fertilization rate in patients after subzonal insemination with spermatozoa lacking outer dynein arms

\begin{tabular}{lrccc}
\hline \multicolumn{3}{c}{ Microinjected oocytes } & \\
\cline { 2 - 4 } Patient & $\begin{array}{c}\text { Number of } \\
\text { oocytes }\end{array}$ & Fertilized & Polyspermic & Pregnancy \\
\hline A1 $^{\mathrm{a}}$ & 10 & 6 & 3 & \\
$\mathrm{A1}^{\mathrm{b}}$ & 16 & 7 & 1 & \\
$\mathrm{~A} 2$ & 10 & 4 & 1 & \\
$\mathrm{~A} 3$ & 11 & 8 & 5 & \\
$\mathrm{~A} 4^{\mathrm{a}}$ & 6 & 1 & 0 & \\
A4 & 11 & 4 & 1 & yes \\
A6 & 9 & 8 & 5 & \\
A7 & 11 & 9 & 4 & \\
A8 & 6 & 5 & 5 & \\
Total & 90 & 52 & 25 & \\
\hline
\end{tabular}

aSecond cycle for the same couple. ${ }^{\text {b}}$ Third cycle for the same couple.

There was no polyploidy among these eggs and no fertilization was obtained in the control IVF group ( 23 oocytes). Three embryo transfers were performed, with one embryo in two cases (patients A1 and A2) and two embryos in the other (patient A3). One pregnancy resulted from the transfer of an embryo but miscarried one month later (patient A2).

In the second series (subzonal insemination only; Table 3), 90 oocytes were microinjected with a greater number of spermatozoa. Fifty-two fertilized $(57.8 \%$ ), of which 25 were polyspermic; the diploid fertilization was obtained for only $30.0 \%$ of the microinjected eggs. Of these diploid zygotes, $87.8 \%$ cleaved normally. Fertilization was obtained in every case in this second series, including patient A4 who had failed to fertilize during the first series. The number of normal embryos obtained for patients who had repeated attempts was generally improved. All the patients had an embryo transfer: four of them with three embryos, and two with one and two embryos, respectively. Two pregnancies were obtained. One gave birth to a healthy baby girl (patient A6), and the other pregnancy is still ongoing (patient A7).

The nuclear status at the time of subzonal insemination was recorded for 80 oocytes. Among metaphase II oocytes $(n=59), 45.8 \%$ fertilized after subzonal insemination. This percentage dropped to $28.5 \%$ for metaphase I oocytes $(n=21)$, but this difference was not statistically significant $(P>0.05)$. No fertilization occurred when less than five spermatozoa were placed in the perivitelline space (Fig. 2). Thirty-seven per cent of the oocytes microinjected with five to nine spermatozoa fertilized with $16.7 \%$ rate of polyspermy. When more than ten and 15 spermatozoa were microinjected, the fertilization rates increased to 46.7 and $47.3 \%$, respectively, with a moderate augmentation of the polyspermy rate $(21 \%)$. A correlation coefficient of 0.81 was found between the fertilization rate for the first attempt of five patients (A1, A2, A3, A5 and A6) whose oocytes were microinjected with the same range number of spermatozoa $(8.5 \pm 3.6)$ and the percentage of hamster oocytes with decondensed sperm heads $(P=0.09)$. Furthermore, the number of spermatozoa that allows a diploid fertilization was

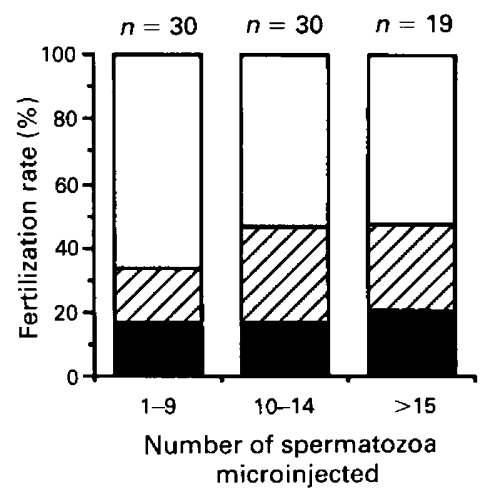

Fig. 2. Fertilization rate in patients according to the number of spermatozoa lacking outer dynein arms microinjected. $\square$ microinjected oocytes; 团 diploid zygotes; polyspermic zygotes.

different from one couple to another, but was remarkably constant for individual couples, from one egg to another and between repeated attempts.

\section{Discussion}

It would be reasonable to query the ethics of assisting fertilization in patients with spermatozoa that lack outer dynein arms considering the potential risk of transmitted disease. However, no connection between the absence of outer dynein arms and genetically transmitted disease has been established, and neither the men who donated spermatozoa, nor male relatives, nor offspring born five years ago after IVF presented with respiratory disease (Spira et al., 1986). Furthermore, no defects in respiratory ciliary cells were found in patients whose spermatozoa lacked outer dynein arms (Escudier et al., 1990). In sea urchins, it has been shown that the alpha-heavy chains of sperm flagellar and ciliary outer arm dyneins are different (Ogawa et al., 1990). It is possible that such a difference also exists in other species. The potential risk of transmitting respiratory diseases, therefore, seems low. However, patients were informed of this possibility, and their acceptance of a long-term follow-up of the children has been obtained.

We report a series of 14 attempts of subzonal insemination with spermatozoa lacking outer dynein arms, including a comparison between subzonal and routine IVF insemination in a prospective randomized clinical trial, yielding three pregnancies and the birth of one baby. Zona-free hamster eggs decondensed human immotile spermatozoa lacking both dynein arms, suggesting that fertilizing ability may be, at least partially, dissociated from the sperm movement pattern (Aitken et al., 1983). These spermatozoa can undergo normal acrosome reaction as seen by transmission electron microscopy (Aitken et al., 1983). Fertilization of three human oocytes by subzonal insemination of a single immotile spermatozoon has also been achieved (Bongso et al., 1989). The embryos obtained cleaved normally but their transfer did not result in pregnancy. Spermatozoa lacking only outer dynein arms can also decondense in zona-free hamster eggs, although the mean percentage of oocytes with swollen sperm heads is lower than that with normal control spermatozoa (Courtot et al., 1985). These data 
demonstrate the ability of these spermatozoa to complete capacitation, to undergo acrosome reaction and to attach to the vitelline membrane and fuse with it (Courtot et al., 1985). Pregnancy with spermatozoa lacking outer dynein arms has been achieved. Spira et al. (1986) carried out an IVF programme of eight cycles for five couples presenting this sperm defect. Out of 36 oocytes, three were fertilized for one patient. The embryo transfer resulted in a twin pregnancy. The two girls are now five years old and appear normal. All these results demonstrate the low fertilization capability of spermatozoa lacking dynein arms but that when fertilization occurs normal embryos can be produced. We confirm the low fertilizing ability of spermatozoa lacking outer dynein arms when placed in contact with the zona pellucida of human oocytes; zygotes were not obtained after the routine IVF insemination of 23 control oocytes. The duration of the sterility of the couples, and the very low fertilization rate obtained by classic IVF procedures justify complementary techniques such as subzonal insemination. Indeed, the overall fertilization rate was $47.1 \%$ after subzonal insemination for 121 oocytes, with a $37.5 \%$ pregnancy rate per patient.

Few characteristics of spermatozoa lacking outer dynein arms seem to be important. In the first randomized series, the fertilization rate was relatively low $(16.1 \%)$ and the rate of polyspermy was nil. In a similar study performed after IVF failure with structurally normal spermatozoa, microinjection of comparable numbers of spermatozoa under the zona pellucida almost doubled the fertilization rate $(29.4 \%)$ and the polyspermy rate was very high (Wolf et al., 1992). This suggests that the fusiogenic capability of spermatozoa lacking outer dynein arms is decreased even when microinjected under the zona pellucida. These findings confirm the observations previously made with the hamster oocyte penetration test. This is why we decided to increase the number of microinjected spermatozoa to more than 10 in the second series. This resulted in an improvement in the fertilization rate (up to $57.8 \%$ ) but with a polyspermy rate of $27.8 \%$. However, the percentage of diploid zygotes was $30.0 \%$ of microinjected oocytes, which is a better result than in the first series. In every attempt of the second series, there was a minimum of one egg fertilized per couple and one embryo transfer, even for patient A4 who failed to achieve fertilization in the first cycle. The difference in the rate of polyspermy between the two series (nil for the first and $27.8 \%$ for the second) is surprising since the increase of the number of spermatozoa microinjected was relatively small. It suggests that the fertilization rate and the rate of polyspermy are not linearly related to the number of spermatozoa microinjected into the perivitelline space. The fertilization rate reached a plateau when more than ten spermatozoa were microinjected and data collected from more than 1000 oocytes after subzonal insemination (not shown) suggest that a threshold might exist.

To reduce polyspermy, it would be useful to have criteria that could predict the optimum number of spermatozoa to microinject. The correlation between the hamster egg penetration assays and the fertilization rate after zona drilling for patients who had previous unsuccessful IVF cycles has been studied but found not to be significant (Vazquez-Levin et al., 1990). We also failed to find any correlation when we performed subzonal insemination for patients with previous IVF failure (Wolf et al., 1992). This correlation coefficient for the five patients who had the same number range of spermatozoa microinjected almost reaches significance in spite of the small number of cases. It could probably serve as a guideline to determine prospectively the number of spermatozoa to microinject. This number would also probably depend upon the percentage of motile and acrosome-reacted spermatozoa present in the suspension after migration, as well as on oocyte quality. This hypothesis is under evaluation.

In conclusion, subzonal insemination appears to be a reliable technique for achieving fertilization and pregnancy in cases of infertility related to flagellar dyskinesia owing to the lack of outer dynein arms and should be preferred to IVF. The zonafree hamster egg penetration assay appears to be a good indicator of the number of spermatozoa to microinject, but this needs to be confirmed by a study involving larger numbers of patients.

This work has been supported by INSERM CJF 8810 and Assistance publique 'Contrat de Recherche Clinique $n^{\circ}$ 910602'. Part of the results were presented at the $7^{\text {th }}$ World Congress on In Vitro Fertilization and Assisted Procreation, Paris, July 1991.

\section{References}

Afzelius BA and Eliasson R (1979) Flagellar mutants in man: on the heterogeneity of the immotile-cilia syndrome Journal of Uttrastructure Research 69 $43-52$

Aitken RJ, Ross A and Lees MM (1983) Analysis of sperm function in Kartagemer's syndrome Fertility and Sterility $40696-698$

Aitken RJ, Warner P and Reid C (1986) Factors influencing the success of sperm-cervical mucus interaction in patients exhibiting unexplained infertility Journal of Andrology 7 3-10

Bacetti B, Burrini AG, Dallai R and Pallini V (1979) The dynein electrophoretic bands in axoneme lacking the inner or the outer arm Joumal of Cell Biology $\mathbf{8 0}$ $334-340$

Biggers JD, Whitten WK and Whittingham DG (1971) The culture of mouse embryos in vitro. In Methods in Mammalian Embryology pp 86-116 Ed. JC Daniel. Freeman, San Francisco

Bongso TA, Sathananthan AH, Wong PC, Ratnam SS, Ng SC, Anandakumar C and Ganatra S (1989) Human fertilization by microinjection of immotile sperm Human Reproduction 4 175-179

Courtot AM, Jeulin C, Escalier D, Serres C, Feneux D and Jouannet P (1985) Ability of human spermatozoa without dynein arms to penetrate zona-free hamster oocytes. In Human in vitro fertilization. INSERM Symposium No. 24, pp 145-148 Eds J Testard and R Frydman. Elsevier Science Publishers, Amsterdam

David G, Bisson JP, Czyglik F, Jouannet P, Gernigon C (1975) Anomalies morphologiques du spermatozoide humain: (I) proposition pour un systeme de classification Joumal de Gynecologie Obstetrique et Biologie de la Reproduction 4 17-36

Escalier D and David G (1984) Pathology of the cytoskeleton of the human sperm flagellum: axonemal and peri-axonemal anomalies Biology of the Cell $\mathbf{5 0}$ 37-52

Escudier E, Escalier D, Pinchon MC, Boucherat M, Bernaudin JF and Fleury-Feith J (1990) Dissimilar expression of axonemal anomalies in respiratory cilia and sperm flagella in infertile men American Review of Respiratory Disease 142 $674-679$

Fishel S, Jackson P, Antinori S, Johnson J, Grossi S and Versaci C (1990) Subzonal insemination for the alleviation of infertility Fertility and Sterility 54 828-835

Frydman R, Forman RG, Belaisch-Allart J, Hazout A, Rainhorn JD, Fries N and Testart J (1988) improvements in ovarian stimulation for in vitro fertilization Annals New York Academy of Sciences 541 30-36

Garrisi GJ, Talansky BE, Grunfeld L, Sapira V, Navot D and Gordon JW (1990) Clinical evaluation of three approaches to micromanipulation-assisted fertilization Fertility and Sterility $\mathbf{5 4} 671-677$ 
Jouannet P, Escalier D, Serres C and David G (1983) Motility of human sperm without outer dynein arms Journal of Submicroscopic Cytology 15 67-71

Jouannet P, Ducot B, Feneux D and Spira A (1988) Male factors and the likelihood of pregnancy in infertile couples. I. Study of sperm characteristic International Journal of Andrology 11 379-394

Mortimer D, Curtis EF, Camenzind AR and Tanaka S (1989) The spontaneous acrosome reaction of human spermatozoa incubated in vitro Human Reproduction 4 57-62

Ng SG, Bongso A, Ratnam SS, Sathananthan H, Chan CLK, Wong PC, Hagglund L, Anandakumar, Wong YC and Goh VHH (1988) Pregnancy after zona transfer of sperm under zona pellucida Lancet 2790

Ogawa K, Yokota E, Hamada Y, Wada S, Okuno M and Nakajima Y (1990) The outer arm dynein alpha-chains of sea urchin sperm flagella and embryonic cilia are different Cell Motility and the Cytoskeleton 16 58-67
Spira A, Jouannet P, Belaisch J, De Almeida M, Jardin A, Testart J, Feneux D, de Mouzon I and Frydman R (1986) In-vitro fertilization and the treatment of male infertility Program of the XII ${ }^{\text {h }}$ World Congress on Fertility and Sterility pp 54. Singapore

Vazquez-Levin M, Kaplan P, Sandler B, Garrisi GJ, Gordon J and Navot D (1990) The predictive value of zona-free hamster egg sperm penetration assay for failure of human in vitro fertilization and subsequent successful zona drilling Fertility and Sterility 53 1055-1059

Veeck LL (1986) Human oocytes at the time of follicular harvest. In Atlas of the Human Oocyte and Early Conceptus pp 5-131 Ed. HW Jones. Williams and Wilkins, Baltimore

Wolf JPh, Ducot D, Kunstmann JM, Frydman R and Jouannet P (1992) Influence of sperm parameters on outcome of subzonal insemination in the case of previous IVF failure Human Reproduction 7 1407-1413 